\title{
Antecedentes organizacionales de la calidad de servicio: un modelo de relaciones estructurales
}

\author{
Organizational antecedents of service quality: a model of structural equations
}

\begin{abstract}
Alejandro Orgambídez-Ramos
Universidade do Algarve, Faculdade de Ciências Humanas e Sociais, Research Centre for Spatial and Organizational Dynamics
\end{abstract} (CIEO), Campus de Gambelas, 8005-139 Faro, Portugal, aoramos@ualg.pt

\section{Maria Helena de Almeida}

Universidade do Algarve, Faculdade de Economia, Research Centre for Spatial and Organizational Dynamics (CIEO) 8005-139 Faro, Portugal, halmeida@ualg.pt

\section{Resumen}

Diferentes modelos de calidad y gestión incluyen las percepciones de los clientes así como las de los gerentes y empleados de contacto. La calidad del servicio no puede ser entendida sin considerar el papel clave jugado por estos últimos. En este sentido, las compañías deben asegurar la gestión adecuada de sus recursos humanos para garantizar la excelencia del servicio. El modo en que los empleados de contacto reaccionan frente a las prácticas organizacionales (p.ej., empowerment organizacional) y al clima organizacional es importante para el desarrollo y comprensión de la calidad del servicio. El objetivo de este estudio es examinar la relación entre el empowerment organizacional, clima organizacional de calidad y calidad del servicio funcional. Y, el efecto mediador del clima organizacional de calidad en la relación entre el empowerment organizacional y la calidad de servicio. Para probar este modelo se han recogido los datos de 428 empleados de contacto de hoteles de Andalucía. El ajuste del modelo y el test de mediación fue realizado mediante un modelo de ecuaciones estructurales con ayuda del programa STATA. Los resultados mostraron que el empowerment organizacional y el clima de calidad fueron predictores significativos de la calidad de servicio funcional. No obstante, el clima de calidad solo medió la relación entre el control sobre las decisiones y la calidad funcional. Las implicaciones teóricas y prácticas del estudio son discutidas.

Palabras clave: empowerment organizacional, clima de calidad, calidad de servicio funcional.

\section{Abstract}

Different models of quality management include customers' perceptions as well as the perception of executives and contact employees. Service quality can hardly be understood without the critical role played by the later. In this sense, companies must make sure they have an adequate management of human resources in order to guarantee such quality. How contact employees react to organizational practices (i.e., organizational empowerment) and organizational climate is important for developing and understanding service quality. The aim of this study is to examine the relationship among organizational empowerment, climate of quality and functional service quality, and the mediator role of the organizational climate of quality in the relationship between the organizational empowerment and the quality of service. To test this model, data was collected from 428 contact employees from Andalusian hotels. Model fit and mediation test was examined using structural equation modeling technique with the help of STATA. Results showed that organizational empowerment and climate for service were significant predictors of functional service quality. However, climate for service did not mediate the relationship between fluidity in information sharing and functional quality. The theoretical and practical implications of this study are discussed.

Keywords: organizational empowerment, climate for quality, functional service quality.

\section{Introducción}

En la actualidad, los cambios económicos, marcados por una mayor competitividad generada por la globalización de la economía internacional, unido a un incremento de las exigencias de los consumidores y un período de crisis a nivel mundial, aconsejan transformaciones profundas en la gestión empresarial del sector turístico. Dentro del sector turístico, los establecimientos hoteleros están experimentando una fuerte competencia. En un escenario caracterizado por la competitividad, la apuesta por la calidad supone una ventaja competitiva difícil de superar, favoreciendo la imagen del servicio que los propios clientes transmiten unos a otros (Peiró, Martínez-Tur, \& Ramos, 1999). Diversos estudios muestran que la percepción de un servicio de baja calidad por parte del cliente es una de las principales razones para que se produzca el cambio a otra empresa de la competencia (Moliner, Carrasco, MartínezTur, \& Marzo, 2004).

Ante esta situación, el estudio de los factores relacionados con la calidad del servicio ha adquirido especial importancia como un elemento de competitividad clave, sobre todo el papel desempeñado por el empleado de contactos (contact employee). Los empleados de contacto son "la imagen" de la empresa, definen el producto y lo promueven directamente hacia el consumidor. En consecuencia, varios autores han propuesto que la existencia de climas y prácticas en las empresas centradas en la calidad pueden afectar positivamente a las actitudes y conocimientos del personal de contacto y, por esta via, en la calidad de servicio percibido por los clientes (MendozaSierra, Orgambídez-Ramos, Borrego-Alés, Gonçalves, \& Santos, 2014a; Salanova, Agut, \& Peiró, 2005; Schneider, Ehrhart, Mayer, Saltz, \& Niles-Jolly, 2005). Por otro lado, el empowerment aparece como una herramienta fundamental de gestión de los empleados, ya que permite la discrecionalidad y la flexibilidad para tomar decisiones que satisfagan las necesidades de los clientes (Mendoza-Sierra, León-Jariego, Orgambídez-Ramos, \& Borrego-Alés, 2009; Mendoza-Sierra, Orgambídez-Ramos, León-Jariego, \& García-Carrasco, 2014b). En este sentido, el objetivo de este estudio es analizar la relación entre el empowerment 
organizacional, clima organizacional de calidad y calidad del servicio funcional. $Y$, el efecto mediador del clima organizacional de calidad en la relación entre el empowerment organizacional y la calidad de servicio. Por lo tanto, este artículo presenta la siguiente estructura: Revisión de la literatura; Metodología (Participantes y procedimiento, Instrumentos Análisis de datos); Resultados (Análisis previos, Descriptivos y correlaciones, Análisis de mediación); Conclusión; e, Referencias.

\section{Revisión de la literatura}

En los últimos años se ha incrementado de manera considerable el interés por la calidad de servicio y la satisfacción del usuario (Cañizares, López, \& Millán, 2007; Wang, Chen, \& Chen, 2012). Esto no es extraño si se tiene en cuenta que la calidad se considera como un factor relevante para explicar la competitividad y la viabilidad de las organizaciones, así como las experiencias positivas de las personas en sus actividades de consumo. La calidad del servicio, a través de la satisfacción que puede generar, influye sobre la lealtad de los usuarios y la prosperidad económica de las organizaciones (Peiró et al., 1999; Sánchez-Hernández, Martínez-Tur, Peiró, \& Ramos, 2009). En consecuencia, los responsables de las organizaciones están interesados en conocer los elementos de la calidad determinantes de las percepciones positivas de los clientes.

Dentro del sector hotelero, la calidad del servicio resulta fundamental para hacer frente con éxito a los nuevos tiempos. Parasuraman, Zeitham y Berry (1988) en su modelo de calidad percibida, además de incluir la perspectiva de los clientes, incluyeron la percepción de los empleados de contacto y de los directivos como agentes igualmente implicados en la calidad del servicio. En consecuencia, los empleados de contacto pueden considerarse como uno de los pilares de la calidad de servicio, especialmente en los establecimientos hoteleros (Moliner et al., 2004). Los establecimientos hoteleros, entre otros, se caracterizan por un algo grado de interacción personal, donde los empleados implicados en el contacto directo con el cliente no sólo representan a la empresa, sino que definen el producto y lo promueven directamente hacia el consumidor (Mendoza-Sierra et al., 2014a; Moliner et al., 2004; Schneider, White, \& Paul, 1998; Schneider et al., 2005). La calidad del servicio ofrecida por los empleados de contacto puede ser clasificada de dos formas: calidad relacional y calidad funcional. Mientras que la calidad relacional se refiere a la empatía y los elementos afectivos de la relación cliente-empleado, la calidad funcional tiene que ver con la eficiencia del trato y del servicio recibido (Sánchez-Hernández et al., 2009).

En la misma línea, las empresas de servicios deberían alcanzar un clima interna que propicie un impacto positivo en las actitudes y en los conocimientos del personal de contacto y, por esta vía, en la calidad de servicio percibida por los clientes (Schneider et al., 1998; Schneider et al., 2005). En otras palabras, los directivos y responsables deben comercializar internamente "una mentalidad de servicio" y gestionar eficazmente las prácticas organizacionales para asegurar que las actitudes $y$ comportamientos del personal de contacto proporcionen un servicio de calidad.

Dentro de las prácticas de recursos humanos más efectivas y relacionadas con el servicio de calidad cabe destacar el empowerment (Hartline \& Ferrell, 1996; Matthews, Diaz, \& Cole, 2003). El empowerment hace referencia a la discrecionalidad de los empleados para tomar decisiones sobre las actividades relacionadas con su trabajo, y tengan comportamientos proactivos en sus trabajos (Bowen \& Lawler, 1992). El empowerment resulta necesario ya que los 'empleados de contacto necesitan flexibilidad para tomar decisiones creativas que proporcionen una satisfacción completa a los clientes' (Hartline \& Ferrell, 1996). A pesar de la necesidad de que, durante los encuentros de servicio, el personal de contacto tenga responsabilidad y autoridad para tomar decisiones "próximas al cliente", muchas empresas tienen establecidos procedimientos rígidos y el personal no tiene capacidad para buscar soluciones que proporcionen el nivel de servicio requerido (Bowen \& Lawler, 1992; Hartline \& Ferrell, 1996).

El empowerment puede darse en diferentes niveles: individual u organizacional. El empowerment organizacional hace referencia a los factores que potencian el empowerment de los trabajadores en cualquier empresa $\mathrm{u}$ organización. Según la propuesta de Matthews, Diaz y Cole (2003), pueden identificarse tres dimensiones del empowerment organizacional: la estructura dinámica, el control sobre las decisiones y la fluidez de la información. La estructura dinámica se refiere a la provisión por parte de la empresa de directrices que faciliten la toma de decisiones de los empleados en sus puestos. El control sobre las decisiones hace referencia al dominio y control del empleado sobre los aspectos relativos a su desarrollo profesional. Finalmente, la fluidez de la información indica la existencia de mecanismos de comunicación de la información necesaria para realizar el trabajo (Matthews et al., 2003; Mendoza-Sierra et al., 2009). El empowerment se ha convertido en una práctica especialmente valorada, resultado de cambios que ocurren en el ambiente de trabajo y que provocan el "aplanamiento" de la estructura organizativa, y de cambios en las creencias sobre el valor estratégico de los trabajadores en las organizaciones, reconocidos como un elemento de ventaja competitiva (Pelit, Yüksel \& Arslantürk, 2011).

Además del empowerment, otra variable que está teniendo un papel igualmente relevante a la hora de comprender la calidad de servicio y la satisfacción de los clientes es el clima organizacional de calidad. El clima organizacional de calidad se define como las percepciones de los empleados acerca de las prácticas, los procedimientos y las conductas que son esperadas y recompensadas por la organización en relación con la calidad (Schneider et al., 1998). En investigaciones previas se han encontrado relaciones positivas entre el clima organizacional asociado a la calidad y las percepciones de calidad de los clientes (Salanova et al., 2005; Schneider et al., 1998; Schneider et al., 2005). Cuanto mayor era el clima percibido, mayores eran también las percepciones de calidad por parte de los clientes y su 
satisfacción con el mismo (Moliner et al., 2004; Schneider et al., 1998; Schneider et al., 2005).

La influencia del clima también se ha observado en las actitudes y comportamientos de los empleados de contacto (Mendoza-Sierra et al., 2014b; Salanova et al., 2005). A este respecto, es más probable que los trabajadores presten un servicio excelente cuando la organización espera y recompensa dicho comportamiento y establece prácticas profesionales que facilitan un servicio de calidad (Bowen \& Lawler, 1992; Schneider et al., 1998; Schneider et al., 2005). Además, se han relacionado las percepciones de clima de servicio de los empleados con resultados organizacionales como la lealtad y la satisfacción de los clientes (Schneider, Salvaggio, \& Subirats, 2002; Sowinski, Fortmann, \& Lezotte, 2008). Así pues, parece evidente que el modo en que los empleados perciben el clima de calidad de su empresa está relacionado con la calidad del servicio percibida por los propios trabajadores pero también por los clientes.

El modo en que los empleados de contacto reaccionan a las prácticas organizacionales basadas en el empowerment y el clima organizacional presente es un elemento de estudio clave para el estudio de la calidad y de las intervenciones necesarias para potenciarla. En este sentido, el objetivo de este estudio es analizar el papel mediador del clima organizacional de calidad entre el empowerment organizacional y la calidad de servicio funcional percibida por los empleados. Concretamente, se plantean las siguientes hipótesis:

H1: El empowerment organizacional tiene una relación positiva y significativa con el clima organizacional de calidad, el cual está relacionado positivamente con la calidad de servicio funcional.

H2: El clima organizacional de calidad media la relación entre el empowerment organizacional y la calidad de servicio funcional.

\section{Metodología}

\section{Participantes y procedimiento}

Se llevó a cabo un estudio ex post facto retrospectivo de un único grupo con múltiples medidas (Montero \& León, 2007), mediante un muestreo de conveniencia en hoteles de playa y ciudad de Andalucía durante el año 2011. El criterio de selección fue pertenecer a puestos de contacto directo, es decir, empleados de recepción y restauración. La muestra quedó compuesta por 428 participantes, de los cuales el $54.44 \%$ son hombres ( $\mathrm{n}=233$ ). El $52.10 \%$ de los empleados se situó entre los 18 y los 29 años. 213 participantes pertenecen a unidades de recepción (49.77\%) y 215 a unidades de restauración (50.23\%). el 42.76\% manifestó tener un contrato fijo, el $42.52 \%$ un contrato temporal y el $14.72 \%$ contratos discontinuos.

La recogida de datos se llevó a cabo a través de un cuestionario aplicado de forma individual y en horario laboral. Los investigadores se desplazaron por los diferentes hoteles y solicitaron a la dirección la participación en el estudio. Conseguida la autorización por parte de los responsables directivos, los cuestionarios fueron aplicados a todos los empleados de contacto que voluntariamente se ofrecieron a participar. Antes de comenzar, se explicaron las instrucciones del cuestionario y se garantizó la total confidencialidad de los datos aportados.

\section{Instrumentos}

Los instrumentos de medición empleados en el estudio se citan a continuación:

Características sociodemográficas y laborales. Se les preguntaba a los participantes por su edad, sexo, nacionalidad, nivel de estudios, número de meses trabajando en el establecimiento hotelero y tipo de contrato -fijo, fijo discontinuo o temporal-.

Empowerment organizacional. Esta variable fue medida con la escala de empowerment organizacional de MendozaSierra et al. (2009). Consta de 19 ítems agrupados en tres dimensiones: estructura dinámica (7 ítems), control de las decisiones (7 ítems) y fluidez de la información (5 ítems). Las respuestas a los ítems fueron dadas usando una escala Likert de 1 (Nada de acuerdo) a 7 (Totalmente de acuerdo). Los coeficientes de fiabilidad obtenidos (Alpha de Cronbach) fueron .87, .78 y .78 para las dimensiones de estructura dinámica, control de las decisiones y fluidez de la información, respectivamente. Estos valores están en la línea de los observados en el trabajo de Mendoza-Sierra et al. (2014b). Altas puntuaciones en la escala indican elevados niveles de empowerment organizacional en la empresa.

Clima organizacional de calidad. Para medir esta variable se utilizó una versión reducida, traducida y utilizada por Moliner et al. (2004) y Salanova et al. (2005). Esta escala consta de 4 ítems cuyas respuestas son dadas en una escala Likert de 7 puntos, que va desde 1 (Totalmente en desacuerdo) hasta 7 (Totalmente de acuerdo). El coeficiente de consistencia interna (Alpha de Cronbach) obtenido fue de .84, similar al valor obtenido en el estudio de MendozaSierra et al. (2014b). Elevadas puntuaciones revelan una mayor presencia de un clima orientado a la calidad.

Calidad de servicio funcional percibida por los empleados. Para medir esta variable se utilizaron 5 ítems de la escala de calidad de servicio funcional de SánchezHernández et al. (2009). Todos los ítems se responden usando una escala de respuesta tipo Likert que va desde 1 (Totalmente en desacuerdo) hasta 7 (Totalmente de acuerdo). El coeficiente de fiabilidad obtenido (Alpha de Cronbach) fue de .87 , coherente con los valores obtenidos en los estudios de Sánchez-Hernández et al. (2009) y de Mendoza-Sierra et al. (2014b). Altas puntuaciones indican la percepción de un servicio prestado de mayor calidad.

\section{Análisis de datos}

Para conseguir los objetivos propuestos, se ha utilizando el paquete estadístico STATA v12. En primer lugar, se ha calculado el test de Harman (Podsakoff, MacKenzie, Lee, \& Podsakoff, 2003) con todos los ítems de las escalas medidas para evaluar el posible impacto de la varianza común del método. Se han realizado análisis descriptivos (media, desviación típica, asimetría), se han obtenido las consistencias internas (Alpha de Cronbach) de las escalas utilizadas y se han calculado las correlaciones (coeficiente de Pearson) entre ellas. 
El sistema de relaciones del modelo de mediación se evaluó un sistema de ecuaciones estructurales basado en correlaciones y tratando las variables como observables (path analysis), utilizando el método de máxima verosimilitud (maximum likelihood). Las variables que se relacionan en el modelo son puntuaciones en test y, por tanto, medidas falibles que contienen error de medida (Acock, 2013). El estudio de la mediación se realizó tomando en cuenta las recomendaciones de Baron y Kenny (1986), utilizando el test de Sobel (1992) para el cálculo de los efectos indirectos.

\section{Resultados}

\section{Análisis previos}

Dado que todos los datos obtenidos están basados en medidas de auto-informe y fueron recogidos en el mismo período de tiempo, la varianza común asociada al método puede sobreestimar o infravalorar las relaciones entre las variables (Podsakoff et al., 2003). El test de Harman (Podsakoff et al., 2003) fue realizado para comprobar el posible efecto de la varianza común. Todos los ítems de las escalas de empowerment organizacional, clima de calidad y calidad funcional fueron sometidos a un análisis exploratorio mediante el método de componentes principales con rotación varimax, forzando la extracción a un solo factor. Si hubiese un problema de varianza asociada al método, el factor extraído debería dar cuenta de más del $50 \%$. Los resultados del análisis exploratorio mostraron un factor que daba cuenta del $31.54 \%$ de la varianza común, por lo que si bien no puede descartarse totalmente el efecto de la varianza común, no parece afectar significativamente a las relaciones entre las variables estudiadas.

\section{Descriptivos y correlaciones}

En la Tabla 1 se muestran las medias, desviaciones típicas, asimetría y correlaciones de las variables del estudio, así como la fiabilidad de las escalas. El clima organizacional y la calidad de servicio funcional obtuvieron valores medios por encima de la media de la escala, al igual que la dimensión estructura dinámica del empowerment organizacional. Por el contrario, las dimensiones control de las decisiones y fluidez de la información registraron valores por debajo de la media.

Tabla 1 - Estadísticos descriptivos y correlaciones de las variables del estudio

\begin{tabular}{|l|c|c|c|c|c|}
\hline & $\mathbf{1}$ & $\mathbf{2}$ & $\mathbf{3}$ & $\mathbf{4}$ & $\mathbf{5}$ \\
\hline 1. Estructura dinámica & 1 & .56 & .58 & .54 & .37 \\
\hline 2. Control sobre decisiones & & 1 & .51 & .53 & .35 \\
\hline 3. Fluidez de información & & & 1 & .44 & .23 \\
\hline 4. Clima de calidad & & & & 1 & .55 \\
\hline 5. Calidad de servicio funcional & & & & & 1 \\
\hline Media & 4.17 & 3.80 & 3.51 & 5.14 & 5.86 \\
\hline Desviación típica & 1.36 & 1.24 & 1.43 & 1.23 & 0.83 \\
\hline Asimetría & -.61 & .67 & .46 & -.59 & -.40 \\
\hline Alpha de Cronbach & .87 & .78 & .78 & .84 & .87 \\
\hline Nota: Todos los coeficientes son estadísticamente significativos (p<.01) & & & \\
\hline
\end{tabular}

Fuente: Elaboración propia.

Los análisis de correlación muestran que, tal y como se esperaba, las dimensiones del empowerment organizacional se relacionaron positivamente $(p<.01)$ con la calidad de servicio funcional, con valores de correlación de Pearson de $.37, .35$ y .23 para estructura dinámica, control de decisiones y fluidez de la información, respectivamente. Igualmente, el clima organizacional de calidad mostró una relación positiva y significativa con la calidad funcional percibida por los empleados $(r=.55, p<.01)$.

\section{Análisis de mediación}

Con el propósito de obtener una representación global de las relaciones entre empowerment organizacional, clima organizacional de calidad y calidad funcional percibida, se ha planteado un modelo de relaciones donde la estructura dinámica, el control de decisiones y la fluidez de la información son variables exógenas y predictoras, el clima organizacional es la variable endógena y mediadora, y la calidad de servicio funcional es la variable endógena resultado. Concretamente, se postula la existencia de un efecto indirecto de las dimensiones del empowerment organizacional sobre la calidad de servicio funcional a través de clima organizacional.

Siguiendo las recomendaciones de Baron y Kenny (1986), para verificar el papel mediador del clima organizacional es necesario que se produzcan tres condiciones. Primero, se debe mostrar que las dimensiones del empowerment organizacional estén relacionadas de forma significativa con el clima organizacional de calidad. Segundo, se debe mostrar que el clima organizacional esté asociado a la calidad de servicio funcional. Tercero, la relación significativa entre las dimensiones del empowerment organizacional y la calidad de servicio funcional quedaría reducida si se controla la influencia del clima de calidad.

Este modelo se ha sometido a comprobación empírica a partir del análisis de ecuaciones estructurales utilizando el método de path analysis. A partir de las recomendaciones de Acock (2013), el análisis se ha realizado siguiendo dos pasos: (1) diseño de un modelo sobre-identificado y (2) rediseño del modelo a partir de los coeficientes significativos encontrados en el paso anterior. En la Figura 1 se presentan los coeficientes estandarizados y los niveles de significación obtenidos en el modelo de ecuaciones estructurales utilizando el método de máxima verosimilitud como procedimiento de estimación de parámetros, así como los valores de varianza explicada $(R 2)$ de las variables clima organizacional y calidad de servicio funcional. El path analysis reveló que el ajuste del modelo final fue adecuado, con un valor de $X^{2}(2)=1.30, p=.52$. Los valores CFI (Comparative Fit Index) y TLI (Tucker-Lewis Index) fueron $1.00 \mathrm{y} 1.01$ 
respectivamente, con un valor SRMR (Standardized Root Mean Square Residual) inferior a .05 (SRMR $=.01$ ) y un valor
RMSEA (Root Mean Square Error of Approximation) de .01 $(90 \% \mathrm{IC}=.01-.09)$ (Hu \& Bentler, 1999).

Figura 1 - Resultados del path analysis. Coeficientes estandarizados y valores de R2 de las variables endógenas

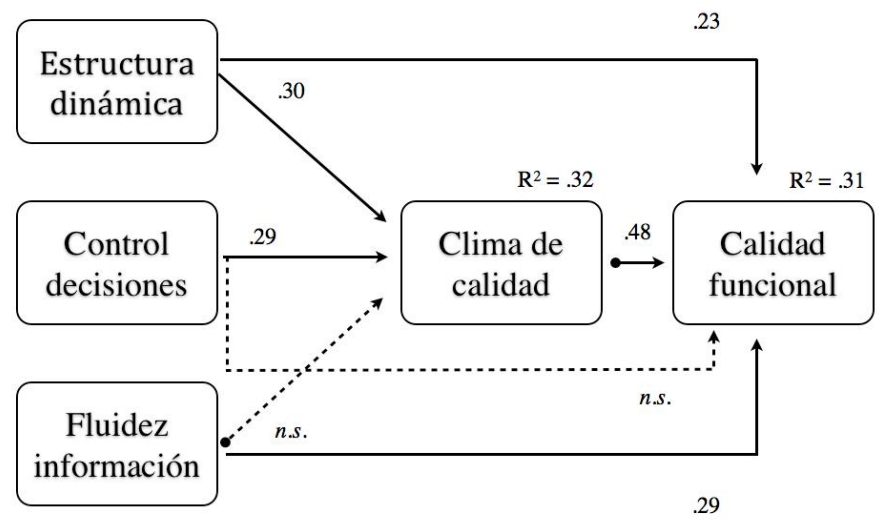

Nota: Todos los coeficientes son estadísticamente significativos $(p<.01)$

Fuente: Elaboración propia.

Resultados del path analysis. Coeficientes estandarizados y valores de $R^{2}$ de las variables endógenas

Como se puede observar, salvo la dimensión fluidez de la información, la estructura dinámica y el control de las decisiones predijeron significativa y positivamente $(p<$ .01) el clima organizacional de calidad, con valores Beta semejantes (.30 y .29 , respectivamente). El total de la varianza del clima de calidad explicado por las dos dimensiones del empowerment organizacional fue del $32 \%$.
Igualmente, la estructura dinámica, la fluidez de la información y el clima de calidad aparecieron como predictores significativos (valores de .23, .15 y .48, respectivamente) de la calidad funcional de servicio ( $p<$ .01 ), explicando un 31\% del total de su varianza. En relación sal papel mediador del clima de calidad, esta variable únicamente medió la influencia del control de las decisiones sobre la calidad funcional. La estructura dinámica tuvo un impacto tanto directo como indirecto, mientras que la fluidez de la información mostró un impacto directo sobre la calidad funcional (ver Tabla 2).

Tabla 2 - Coeficientes estandarizados de los efectos directos, indirectos y totales de empowerment organizacional y clima de calidad sobre calidad funcional de servicio

\begin{tabular}{|c|c|c|c|c|}
\hline Resultado & Efecto Directo & Efecto Indirecto & $z$ & Efecto total \\
\hline $\begin{array}{l}\text { Clima de calidad } \\
\text { Estructura dinámica } \rightarrow \\
\text { Control sobre decisiones } \rightarrow\end{array}$ & $\begin{array}{l}.30^{\mathrm{A}} \\
.29\end{array}$ & $\begin{array}{l}--- \\
---\end{array}$ & & $\begin{array}{l}.30 \\
.29\end{array}$ \\
\hline $\begin{array}{l}\text { Calidad funcional } \\
\text { Estructura dinámica } \rightarrow \\
\text { Control sobre decisiones } \rightarrow \\
\text { Fluidez de información } \rightarrow \\
\text { Clima de calidad } \rightarrow\end{array}$ & $\begin{array}{l}.23 \\
--- \\
.15 \\
.48\end{array}$ & $\begin{array}{l}.15 \\
.14 \\
--- \\
---\end{array}$ & $\begin{array}{l}3.83 \\
3.75\end{array}$ & $\begin{array}{l}.38 \\
.14 \\
.15 \\
.48\end{array}$ \\
\hline
\end{tabular}

Nota: Todos los coeficientes son estadísticamente significativos $(p<.01)$

Fuente: Elaboración propia.

Del efecto total de la estructura dinámica sobre la calidad funcional, el $60.5 \%(.23 / .38=.605)$ fue directo mientras que el $39.5 \%(.15 / .38=.395)$ se dio a través del clima organizacional. En el caso de la fluidez de la información, el efecto no fue mediado por el clima (Beta $=.15$, p <.01); por el contrario, el impacto del control de las decisiones sí estuvo mediado totalmente por el clima organizacional (Beta $=.14, \mathrm{p}<.01)$.

\section{Conclusión}

El objetivo de este estudio fue comprobar el papel mediador del clima organizacional de calidad entre el empowerment organizacional y la calidad de servicio funcional percibida por los empleados de contactos. Los resultados obtenidos solo confirman el papel mediador del clima en el caso de la dimensión control sobre las decisiones. La estructura dinámica tuvo un impacto tanto directo como indirecto sobre la calidad, y la fluidez de la información sólo afectó directamente a la calidad funcional.

La calidad de servicio funcional se refiere a la eficacia en la provisión del servicio (Sánchez-Hernández et al., 2009). En este sentido, los resultados observados confirman la necesidad de una comunicación eficaz que permita hacer llegar la información a los empleados sobre los criterios de la calidad de servicio, junto con directrices que faciliten la toma de decisiones para satisfacer las necesidades de los clientes. Igualmente, la fluidez de la información y la estructura dinámica aparecen como elementos organizacionales clave para la provisión de la calidad. La presencia de estos elementos generan mayores sentimientos de eficacia en los empleados, en el sentido de que saben cuáles son sus tareas y qué se espera de ellos. Estos resultados son coherentes con la literatura existente (Mendoza-Sierra et al., 2014b; Salanova et al., 2005; 
Sowinski et al., 2008; Sánchez-Hernández et al., 2009), donde se ha observado que variables organizacionales asociadas a la comunicación organizacional y a la existencia de guías de actuación se han asociado a las conductas y actitudes de los empleados, y a una mayor percepción de la calidad de servicio por parte de los clientes. Desde un punto de vista organizacional, el estudio confirma la importancia del empowerment en el proceso de construcción de una cultura de calidad total, en particular en el contexto de un entorno empresarial altamente competitivo. Estas prácticas de empowerment organizacional deben planearse e implementarse desde la dirección de la empresa para ser efectivas (Campos, Silva, Mendes, \& Valle, 2014).

El clima organizacional de calidad ha tenido un impacto directo sobre la calidad y ha actuado como mediadora en la relación entre control sobre las decisiones y la calidad funcional. La existencia de un clima centrado en la calidad permite que todos los empleados perciban que la calidad es importante y qué se espera de ellos, por parte de la empresa, en términos de excelencia del servicio. Por otro lado, indica qué conductas son recompensadas y orienta a los empleados sobre los elementos relativos a su desarrollo profesional (i.e., tipo de competencias necesarias). Los aspectos relativos al desarrollo profesional control sobre las decisiones- son mediados por el clima de calidad, puesto que el clima indica cuáles son los aspectos asociados a la calidad del servicio. Estos resultados son consistentes con los estudios de Hartline y Ferrell (1996) y Mendoza et al. (2014b). En este sentido, Wallace, Johnson, Mathe y Paul (2011) expresan que no basta con tener empleados empowered, sino que es necesario que sean conscientes de lo que se espera de ellos en la organización.

Los resultados observados en este estudio deben interpretarse teniendo en cuenta algunas limitaciones. En primer lugar, el diseño transversal invalida las conclusiones de causalidad sobre las relaciones entre variables, si bien la evidencia empírica se construye sobre modelos planteados en estos términos (MacKinnon, Fairchild, \& Fritz, 2007). Otro aspecto tiene que ver con el hecho de que todas las variables del estudios se hayan medido mediante autoinformes, lo que incrementa el riesgo de varianza del método común. Si bien el test de Harman (Podsakoff et al., 2003) indica que la varianza del método común no parece ser un factor relevante, no se puede asegurar totalmente su falta de influencia en las relaciones entre las variables. Por otro lado, sería necesario realizar nuevas investigaciones que permitieran relacionar el empowerment y el clima organizacional con la percepción de la calidad de los clientes, así como la inclusión de variables individuales (i.e., autoeficacia).

Finalmente, puede concluirse que la calidad funcional en los hoteles está determinada en gran parte por características organizacionales ligadas al empowerment organizacional y por la presencia de un fuerte clima organizacional centrado en la calidad. Precisamente, factores como el apoyo de la alta dirección, la comunicación, y la involucración de los empleados son factores clave para obtener unos mejores resultados en la innovación y la calidad del servicio hotelero (Pascual Fernández, Santos Vijande \& López-Sánchez, 2014). La comprensión de los mecanismos subyacentes a estas relaciones debe ser considerada como una prioridad para los establecimientos hoteleros. Ello permitiría el diseño de intervenciones que favorezcan la toma de decisiones, la circulación adecuada de las informaciones así como las conductas esperadas de los empleados relativos a la excelencia en el servicio.

\section{Referencias}

Acock, A. C. (2013). Discovering structural equation modeling using stata. Texas, US: StataCorp LP.

Baron, R. M., \& Kenny, D. A. (1986). The moderator-mediator variable distinction in social psychological research: Conceptual, strategic, and statistical considerations. Journal of Personality and Social Psychology, 51(6), 1173-1182. doi:10.1037/00223514.51.6.1173

Bowen, D., \& Lawler, E. (1992). The empowerment of service workers: What, why, how, and when? Sloan Management Review, 33, 31-39.

Campos, A. C., Mendes, J. C., Silva, J. A. \& Valle, PO.(2014) Critical success factors for a total quality culture: A structural model Fatores críticos de sucesso de uma cultura da qualidade total: um modelo estrutural. Tourism \& Management Studies, 10(1), 7-15.

Cañizares, S., López, J., \& Millán, G. (2007). Análisis de la satisfacción laboral en los establecimientos hoteleros: Un estudio de caso. Tourism \& Management Studies, 3, 125-133.

Hartline, M. D., \& Ferrell, O. C. (1996). The management of customer-contact service employees: An empirical investigation. Journal of Marketing, 60, 52-70. doi:10.2307/1251901

Hu, L., \& Bentler, P. M. (1999). Cutoff criteria for fit indexes in covariance structure analysis: Conventional criteria versus new alternatives. Structural Equation Modeling: A Multidisciplinary Journal, 6(1), 1-55. doi:10.1080/10705519909540118

MacKinnon, D., Fairchild, A., \& Fritz, M. (2007). Mediation analysis. Annual Review of Psychology, 58(2), 593-618.

Matthews, R. A., Diaz, W. M., \& Cole, S. G. (2003). The organizational empowerment scale. Personnel Review, 32, 297-318. doi:10.1108/00483480310467624

Mendoza-Sierra, M. I., León-Jariego, J. C., Orgambídez-Ramos, A., \& Borrego-Alés, Y. (2009). Evidencias de validez de la adaptación española de la organizational empowerment scale [Validity evidence of the Spanish adaptation of the organizational empowerment scale]. Revista de Psicología del Trabajo y de las Organizaciones, 25(1), 17-28.

Mendoza-Sierra, M. I., Orgambídez-Ramos, A., León-Jariego, J. C., \& García-Carrasco, A. M. (2014). Service climate as a mediator of organizational empowerment in customer-service employees. The Spanish Journal of Psychology, 17(E4), 1-10. doi:10.1017/sjp.2014.4

Moliner, C., Carrasco, H., Martínez-Tur, V., \& Marzo, J. C. (2004). Diferencias en clima de servicio y conductas extra-rol hacia el cliente: Una aproximación contextual en hoteles [Climate for service and extra-role customer service differences: A contextual approach in hotels]. Revista de Psicología Social Aplicada, 14, 73-85.

Montero, I., \& León, O. G. (2007). A guide for naming research studies in Psychology. International Journal of Clinical and Health Psychology, 7, 847-862.

Parasuraman, A., Zeithaml, V. A., \& Berry, L. L. (1988). Servqual: A multiple-item scale for measuring consumer perceptions of service quality consumer perceptions of service quality. Journal of Retailing, 64, 12-40.

Pascual-Fernández, P., Santos-Vijande, M. \& López-Sanchez, J. A. (2014) Factores determinantes en el éxito del desarrollo del nuevo servicio [Determinants of success in new service development]. Tourism \& Management Studies, 10(2), 67-74.

Peiró, J. M., Martínez-Tur, V., \& Ramos, J. (1999). El triángulo de la calidad de servicio: Una aproximación psicosocial [The service quality triangle: A psychosocial approach]. Papeles del Psicólogo, 74, 1-12.

Pelit, E., Yüksel, Ö. \& Arslantürk, Y. (2011). The effects of employee empowerment on employee job satisfaction: a study on hotels in Turkey. International Journal of Contemporary Hospitality Management, 23(6), 784-802. 
Podsakoff, P., MacKenzie, S., Lee, J., \& Podsakoff, N. (2003). Common method biases in behavioral research: A critical review of the literature and recommended remedies. Journal of Applied Psychology, 88(5), 879-903.

Salanova, M., Agut, S., \& Peiró, J. M. (2005). Linking organizational resources and work engagement to employee performance and customer loyalty: The mediation role of service climate. The Journal of Applied Psycholgy, 90(6), 1217-1227. doi:10.1037/00219010.90.6.1217

Sánchez-Hernández, R. M., Martínez-Tur, V., Peiró, J. M., \& Ramos, J. (2009). Testing a hierarchical and integrated model of quality in the service sector: Functional, relational, and tangible dimensions. Total Quality Management \& Business Excellence, 20, 1173-1188. doi:10.1080/14783360903247577

Schneider, B., Ehrhart, M. G., Mayer, D. M., Saltz, J. L., \& Niles-Jolly, K. (2005). Understanding organization-customer links in service settings. The Academy of Management Journal, 48, 1017-1032. doi:10.5465/AMJ.2005.19573107

Schneider, B., Salvaggio, A. N., \& Subirats, M. (2002). Climate strenght: A new direction for climate research. Journal of Applied Psychology, 87, 220-229. doi:10.1037/0021-9010.87.2.220

Schneider, B., White, S. S., \& Paul, M. C. (1998). Linking service climate and customer perceptions of service quality: Test of a causal model. Journal of Applied Psychology, 83, 150-163. doi:10.1037/0021-9010.83.2.150

Sobel, M. E. (1992). A symptotic confidence intervals for indirect effects in structural equation models. In S. Leinhardt (Ed.), Sociological Methodology (pp. 290-312). Washington, DC: American Sociological Association.

Sowinski, D. R., Fortmann, K. A., \& Lezotte, D. V. (2008). Climate for service and the moderating effects of climate strenght on customer satisfaction, voluntary turnover, and profitability. European Journal of Work and Organizational Psychology, 17, 73-88.

Wallace, J. C., Johnson, P. D., Mathe, K., \& Paul, J. (2011). Structural and psychological empowerment climates, performance, and the moderating role of shared felt accountability: A managerial perspective. Journal of Applied Psychology, 96, 840-850.

Wang, C. H., Chen, K. Y., \& Chen, S. C. (2012). Total quality management, market orientation and hotel performance: the moderating effects of external environmental factors. International Journal of Hospitality Management, 31, 119-129. 